\title{
Inconsistent evidence for the use of tricyclic antidepressants in the treatment of temporomandibular joint disorders
}

\author{
Abstracted from \\ Cascos-Romero J, Vázquez-Delgado E, Vázquez-Rodríguez E, Gay-Escoda C. \\ The use of tricyclic antidepressants in the treatment of temporomandibular joint disorders: \\ systematic review of the literature of the last 20 years. \\ Med Oral Patol Oral Cir Bucal 2009; 14: E3-E7 \\ Address for correspondence: Professor Cosme Gay Escoda, Centro Médico Teknon, \\ C/ Vilana n 12, 08022 Barcelona, Spain. E-mail: cgay@ub.edu
}

\section{Question: Are tricyclic antidepressants effective in the treatment of temporomandibular joint disorders?}

Data sources Medline and the Cochrane Library were consulted to find evidence in the literature.

Study selection The English language studies identified were considered and stratified according to the Strength of Recommendation Taxonomy (SORT) criteria. ${ }^{1}$

Data extraction and synthesis A qualitative review was conducted. Results 11 articles were identified, seven of which were reviews. Conclusions There is evidence, based on inconsistent or limited-quality patient-oriented data (SORT level B recommendation), for the use of tricyclic antidepressants (TCA) in the treatment of temporomandibular joint disorders (TMD).

\section{Commentary}

In 1987, the International Headache Society developed a first edition of the Classification and Diagnostic Criteria for Headache Disorders, Cranial Neuralgias and Facial Pain. ${ }^{2}$ Through the effort of the American Academy of Orofacial Pain, the group of conditions comprising TMD was included in this medical, diagnostic classification. Many disease entities in the oral and facial region, including TMD, present with similar pain patterns. Since the majority of these are medical conditions, dentists must consider diseases unrelated to the masticatory system and include them in their differential diagnosis. As well as this, it is difficult to correctly diagnose orofacial pain, particularly because of the complex interrelationship of somatic (Axis I) and psychosocial (Axis II) factors in the aetiology of these and general chronic pain disorders. As well as somatic factors, psychosocial factors, eg, anxiety, depression and stress, considerably contribute to the patient's pain(s). Okeson consequently states that a treatment plan, based on the correct diagnosis, must include collaboration with a mental health professional. ${ }^{3,4}$

For the treatment of TMD, many modalities have been proposed. Included among these are occlusal splints, physiotherapy, relaxing appliances and pharmacological interventions. ${ }^{5}$ This systematic review discusses the use of TCA in the treatment of TMD using the SORT criteria. The article concludes that the evidence is inconsistent, of limited quality or patient-oriented, resulting in a SORT level $\mathrm{B}$ recommendation.
On close inspection, TMD is not a diagnosis. Myofacial pain and fibromyalgia-examples of musculoskeletal dysfunctions-are TM diagnoses. Other concomitant disorders, based upon psychosocial factors, may also be present.

Given the level of evidence of literature summarised in this review, TCA would not be the first choice with which to start treatment in people with a TM diagnosis: When, after a comprehensive interdisciplinary, somatic examination, a specific TM-diagnosis is in doubt, a mental health professional must be consulted along with other disciplines when making the definitive treatment plan. An interdisciplinary team may, however, conclude that TCA are a fair treatment option for a specific TM-diagnosis. Therefore the relevance of this systematic review for the general practitioner is that they should refer to an orofacial pain specialist or similar oriented clinic, when a more complex disorder is suspected.

Aad JJ Zonnenberg

Prosthodontist, Santpoort-Noord, The Netherlands

1. Ebell MH, Siwek J, Weiss BD, et al. Strength of recommendation taxonomy (SORT): a patient-centered approach to grading evidence in the medical literature. Am Fam Physician 2004; 69: 548-56

2. Classification and diagnostic criteria for headache disorders, cranial neuralgias and facial pain. Headache Classification Committee of the International Headache Society. Cephalalgia. 1988;8 Suppl 7:1-96.

3. Okeson JP (Ed). Orofacial Pain. Guidelines for Assessment, Diagnosis, and Management. Chicago: Quintessenz; 1996.

4. Okeson JP. Management of Temporomandibular Disorders and Occlusion. 6th Edn. St Louis: Mosby; 2008.

5. Al-Ani MZ, Davies SJ, Gray RJM, Sloan P, Glenny AM. Stabilisation splint therapy for temporomandibular pain dysfunction syndrome. Cochrane Library; 2004. issue no 1

Evidence-Based Dentistry (2009) 10, 56. doi:10.1038/sj.ebd.6400656 